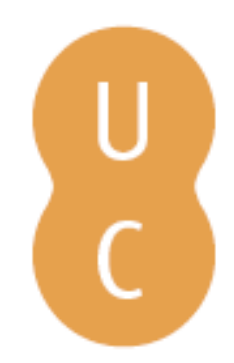

\title{
nommalina
}

\section{Il de fraterno amore di Plutarco tra Thomas Naogeorgus, Ludovicus Russardus e Stephanus Niger}

Autor(es): $\quad$ Tanga, Fabio

Publicado por: Imprensa da Universidade de Coimbra; Annablume

URL

persistente: URI:http://hdl.handle.net/10316.2/41415

DOI: $\quad$ DOI:https://doi.org/10.14195/978-989-26-1306-2_1

Accessed : $\quad$ 26-Apr-2023 09:58:41

A navegação consulta e descarregamento dos títulos inseridos nas Bibliotecas Digitais UC Digitalis, UC Pombalina e UC Impactum, pressupõem a aceitação plena e sem reservas dos Termos e Condições de Uso destas Bibliotecas Digitais, disponíveis em https://digitalis.uc.pt/pt-pt/termos.

Conforme exposto nos referidos Termos e Condições de Uso, o descarregamento de títulos de acesso restrito requer uma licença válida de autorização devendo o utilizador aceder ao(s) documento(s) a partir de um endereço de IP da instituição detentora da supramencionada licença.

Ao utilizador é apenas permitido o descarregamento para uso pessoal, pelo que o emprego do(s) título(s) descarregado(s) para outro fim, designadamente comercial, carece de autorização do respetivo autor ou editor da obra.

Na medida em que todas as obras da UC Digitalis se encontram protegidas pelo Código do Direito de Autor e Direitos Conexos e demais legislação aplicável, toda a cópia, parcial ou total, deste documento, nos casos em que é legalmente admitida, deverá conter ou fazer-se acompanhar por este aviso.

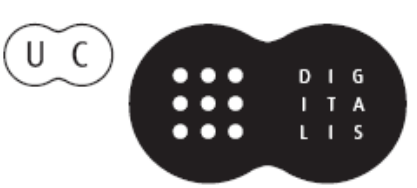




\section{Plutarque}

\section{Éditions, Traductions, Paratextes}

Françoise Frazier, Olivier Guerrier (coords.)

IMPRENSA DA UNIVERSIDADE DE COIMBRA 


\title{
Il De fraterno amore di Plutarco tra Thomas Naogeorgus, Ludovicus Russardus e Stephanus Niger (Plutarch's De fraterno amore in Thomas Naogeorgus, Ludovicus Russardus and Stephanus Niger)
}

\author{
FABio TANGA (tangafabio@libero.it) \\ Università di Salerno
}

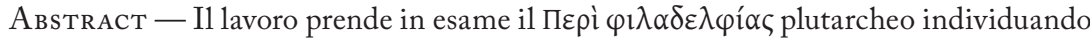
il contributo delle traduzioni latine di XVI secolo allo studio, all'edizione, all'esegesi e alla divulgazione del testo del trattato. Indagando sull'attività versoria del giureconsulto francese Louis Rousard e dell'umanista bavarese Thomas Kirchmeyer nei confronti del De fraterno amore, si delinea una grande attenzione a livello europeo per l'opuscolo plutarcheo, i cui temi e stilemi sono stati ripresi ed imitati anche dal De fraterna benevolentia del filologo cremonese Stefano Negri.
\end{abstract}

Parole chiave - Plutarco; De fraterno amore; L. Rousard; T. Kirchmeyer; S. Negri

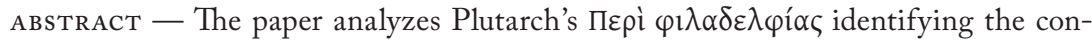
tribution of the sixteenth-century Latin translations to the study of the text of the Plutarchean essay. Investigating the Latin translations of Plutarch's De fraterno amore written by the French jurist Louis Rousard and by the Bavarian humanist Thomas Kirchmeyer, and looking at De fraterna benevolentia composed by the Cremonese philologist Stefano Negri, the paper shows also the reception of Plutarch's Пвpi $\varphi \imath \lambda \alpha \delta \varepsilon \lambda \varphi i^{\prime} \alpha \varsigma$ in sixteenth-century Europe.

KeYwords - Plutarch; De fraterno amore; L. Rousard; T. Kirchmeyer; S. Negri

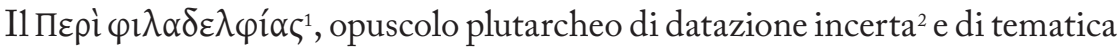
ibrida tra la famiglia e l'amicizia ${ }^{3}$ dedicato ai fratelli romani Avidio Nigrino e Quieto $^{4}$, riesce a coniugare una materia riconducibile alla tradizione filosofica ${ }^{5}$ con l'esperienza personale di Plutarco, marito e padre dotato di eccellente $\varphi \imath \lambda i ́ \alpha$

${ }^{1}$ Classificato al nr. 98 del Catalogo di Lampria, al nr. 13 dell'edizione planudea e al nr. 31 nelle edizioni a stampa dei Moralia; cf. Treu 1873: 11; Irigoin 1987: 314; Postiglione 1991: 9; Pohlenz 2001: 221.

${ }^{2}$ Brokate riteneva il De fraterno amore successivo al De adulatore et amico, al De amicorum multitudine e alla Vita di Catone, mentre Dumortier ha ricondotto l'opuscolo agli ultimi anni del regno di Traiano, datandolo verso il 115 d.C.; cf. Brokate 1913 e Dumortier 1975: 137. Il terminus post quem per la composizione dell'opuscolo potrebbe essere la menzione di un non

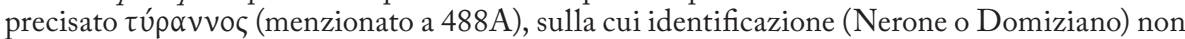
si è concordi; cf. Jones 1966: 70-71 e Postiglione 1991: 10. L'opera sarebbe stata comunque composta nel lasso temporale tra il 68 e il 107 d.C.

${ }^{3}$ Cf. Ziegler 1965: 200.

${ }^{4}$ Sulla corretta identificazione di Quieto cf. Postiglione 1991: 10; 113, n. 3.

${ }^{5}$ Cf. Dumortier 1975: 133-141. 
nei sentimenti e nei rapporti interpersonali ${ }^{6}$. Il De fraterno amore ${ }^{7}$ ha riscosso una grande fortuna in ambito europeo nella seconda metà del XVI secolo, come testimoniato dall'opera di uomini di cultura, attivi in Germania, Francia ed Italia, che può apportare degli utili contributi allo studio di alcuni punti dibattuti del testo plutarcheo.

\section{Il De Fraterno amore di Thomas NaOgeorgus}

A partire dalla città di Straubing, fino a giungere in Turingia e nella Germania centrale a Stoccarda, fu attivo il frate domenicano tedesco, poi parroco e pastore luterano bavarese Thomas Kirchmaier ${ }^{8}$ (nome latinizzato in Thomas Naogeorgus ${ }^{9}$ ), divenuto celebre nella sua epoca quale infiammato teologo protestante, fine drammaturgo e dotto pamphlettista. Noto per la satira anti-cattolica intitolata Regnum papisticum (1553) e celebrato per la composizione di tragedie quali il Pammachius (1538), gli Incendia seu Pyrgopolinices (1541) ed il Mercator seu iudicium (1540), Naogeorgus fu una figura di spicco del teatro tedesco della seconda metà del 1500 e si distinse fin da giovane per la sua cultura classica ed umanistica ed il suo fermento religioso. Oltre ad aver tradotto, tra il 1552 ed il 1558, altre opere di Sofocle, Isocrate, Giovanni Crisostomo e Sinesio ${ }^{10}$, nel 1555 Kirchmaier pubblicò a Basilea, presso l'editore Ioannes Oporinus, la traduzione latina ${ }^{11}$ di sette opuscoli dei Moralia plutarchei ${ }^{12}$, tra cui il De fraterno amore, preceduta da una epistola con dedica rivolta al "generosus ac nobilis dominus" protestante Huldricus Fugger ${ }^{13}$, signore di Kirchperg e Weissehorn, membro di una

${ }^{6}$ Cf. Postiglione 1991: 24.

${ }^{7}$ Titolo tradotto con Commentatio de fraterno amore da T. Kirchmaier e Commentarius de Fraterna amicitia da L. Rousard.

${ }^{8}$ Sulla figura di Thomas Kirchmaier (1508-1563) cf. Shade 1863: 328; Hübner 1913: 297-338; Hübner 1920: 193-222; Theobald 1931: 143-165; Levinger 1935: 145-166; Roloff 1979: 455-475; Krojer 2006; Volpe Cacciatore 2010: 459-466.

${ }^{9}$ Thomas Kirchmaier fu noto anche con le varianti onomastiche di Thomas Kirchmeyer, Kirchmayer, Kyrchmayer, Kirchmair, Kirchmayr, Kirchmeier, Kyrchmayr o Kirchbauer; Thomas Neageorgius o Naogeorg; Thomas Naogeorgus Kirchmayer; Thomas Nageorgus, Nageorgius o Naogeorgius; Thoma Naogeorgo, Naogeorgus o Naogeorgius Straubingensis; Thomas Neubaur; con gli pseudonimi di Thomas Pfarrkircher, Hubelschmeiser, Neübaur, Neubauer o Neumeyer, oltre che con l'abbreviazione Th. N. S.

${ }^{10}$ Cf. Volpe Cacciatore 2010: 459, n. 2.

${ }^{11}$ Intitolata: Plutarchi Chaeronensis, Summi Philosophi, Libelli septem, in Latinum conversi, cum antea versi non essent: Quorum catalogum versa pagina invenies. Thoma Naogeorgo Straubingensi interprete. Basileae, per Ioannem Oporinum, 1556; cf. Naogeorgus 1556.

${ }^{12}$ I sette opuscoli plutarchei tradotti in latino sono: Septem Sapientium convivium; De superstitione; Quomodo se quispiam citra invidiam laudare possit; De fraterno amore; De garrulitate; De syllaba ei apud Delphos e De Socratis damonio; cf. Naogeorgus 1556.

${ }_{13}$ Ulrich Fugger fu attivo ad Augsburg, Bologna, Roma e Bourges. Nel 1567 si trasferì ad Heidelberg, e dopo la sua morte, la sua biblioteca personale fu collocata nella Chiesa dello 
famiglia di mercanti e banchieri originaria di Augsburg. Naogeorgus appronta

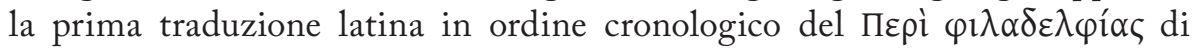
Plutarco, e la novità dell'impresa è annunciata fin dal frontespizio dell'opera, dove si ricorda che i "Libelli septem" sono stati “... in Latinum conversi, cum antea versi non essent”. Poi, tale consapevolezza dell'originalità del proprio lavoro riappare anche all'interno dell'epistola dedicatoria, datata 23 agosto 1555, dove l'autore spera di produrre "aliquid utilitatis \& delectationis" per il lettore, essendosi dedicato, tra gli opuscoli plutarchei, alla traduzione di “... ea quae nondum quidem (quantum ego sciam) conversa essent”. L'epistola dedicatoria del volumetto di traduzioni offre anche altri numerosi elementi degni di attenzione. L'autore afferma di essersi imbattuto in un “... Graecus codex Moralium Plutarchi” e di esser rimasto "delectatus" dalla lettura dello stesso, oltre che "admiratus" per il "divinum ingenium" dello scrittore di Cheronea, che dimostra di essere un bravo filosofo, un grande conoscitore degli scrittori antichi a lui precedenti e di innumerevoli storie ed aneddoti citati a profusione, ed un eclettico nel trattare "feliciter" gli argomenti più svariati. Ammirando la "absoluta eruditio", la "probitas", la "doctrina efficax, sana \& fructuosa", la "integra mens" ed i "probi atque humani mores" del Cheronese, Naogeorgus gli riconosce argomenti che "... Christiani hominis putes" ${ }^{14}$ e lo descrive come splendidamente beneficiato dalla elargizione dei doni della "divina bonitas". Il contenuto dei trattati dei Moralia tradotti da Kirchmeier è definito, poi, di estremo interesse per il lettore in quanto privo di "spinosi labyrinthi" e provvisto di "utilitas rerum" e "pondus sententiarum". Quindi Naogeorgus esprime un giudizio sulla propria attività versoria, esponendone i limiti linguistici e stilistici e spiegandone gli obiettivi letterari e culturali. Il traduttore ammette di non aver potuto “... pari elegantia \& gratia reddere" il contenuto degli opuscoli, sostiene di aver rifiutato di “... longius a Graeco digredi" e di aver tradotto il testo greco "astrictius". Prendendo le distanze da altri traduttori, l'autore mostra consapevolezza delle difficoltà sottese all'operazione di mediazione e trasferimento culturale intrinseca alla traduzione dal greco al latino, affermando: "Non enim paraphrastes esse studui (quod nonnulli solent) sed interpres". Le discettazioni plutarchee sulla tematica dell'amore fraterno richiamarono senza dubbio l'attenzione del teologo e pastore protestante Thomas Kirchmeyer, che si avvicinò allo scrittore

Spirito Santo come parte della Biblioteca Palatina. In merito ad Ulrich (1526-1584) e alla famiglia dei Fugger di Augsburg cf. Strieder 1931; Kluger 2009; Häberlein 2012.

${ }^{14}$ L'interesse del luterano Kirchmeyer per il De fraterno amore plutarcheo forse è ascrivibile proprio a quella "dolcezza, serietà, efficacia e partecipazione ... al confine tra l'etica pagana e l'etica cristiana" (cf. Postiglione 1991: 24-25) che Plutarco, così sensibile ai valori della famiglia, aveva dimostrato fin dalla Consolatio ad uxorem. Già J. De Romilly aveva parlato della “douceur” di Plutarco; cf. De Romilly 1979: 293. 
di Cheronea per la sua saggezza e moderazione affini alla temperie cristiana e con una certa consapevolezza intellettuale delle difficoltà tecniche e culturali dell'opera intrapresa.

La traduzione di Naogeorgus apporta un contributo molto utile alla comprensione del testo del De fraterno amore plutarcheo in uno dei loci che l'edizione della Bibliotheca Teubneriana a cura di M. Pohlenz ha giudicato lacunosi, ovvero il passo 491D, dove Plutarco esorta ad avere estrema cura non solo dei fratelli, ma anche della loro famiglia e dei loro figli, mostrando un'attenzione particolare nei confronti della moglie del fratello. In particolare, prendendo in considerazione 491D6-8 ${ }^{15}$ :

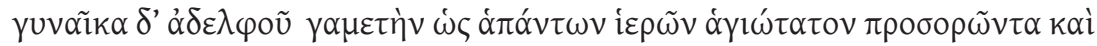

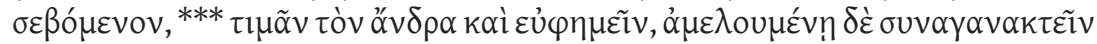
$\chi \alpha \lambda \varepsilon \pi \alpha i ́ v o v \sigma \alpha v \delta \varepsilon \grave{\varepsilon} \pi \rho \alpha u ̈ v \varepsilon ı v$

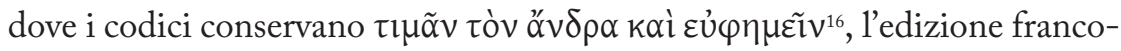
fortana del 1599 ${ }^{17}$, seguita da Reiske ${ }^{18}$, Wyttenbach ${ }^{19}$, Bernardakis ${ }^{20}$ e Postiglio-

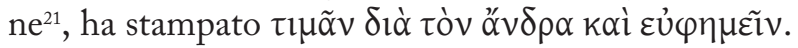

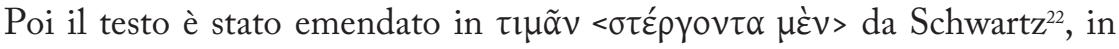

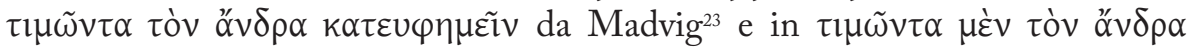
$\kappa \alpha \tau \varepsilon \cup \varphi \eta \mu \tilde{\imath} v$ da Helmbold ${ }^{24}$, mentre Pohlenz ${ }^{25}$ ha proposto in apparato

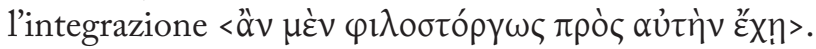

I traduttori hanno effettuato delle scelte differenti, in quanto la versio gallica di Amyot $^{26}$ recita "... la tenir \& reverer comme une relique tressaincte, pour l'amour de son mari, la louër" ed il volgarizzamento di Adriani ${ }^{27}$ traduce “... l'osservare e riverire in guisa di reliquia santissima la moglie del fratello, ed

${ }^{15}$ Il testo proposto è quello pubblicato da Pohlenz 2001: 252.

${ }^{16} \mathrm{Il}$ testo dei manoscritti è accolto dall'editio basileensis; cf. Basilea 1542: 418.

${ }^{17}$ Wechel 1599: 419.

${ }^{18}$ Cf. Postiglione 1991: 102.

${ }^{19}$ Wyttenbach 1796: 998.

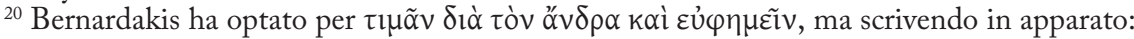
“ $\delta i \grave{\alpha}]$ om. mei codd.”; cf. Bernardakis 1891: 275.

${ }^{21}$ Cf. Postiglione 1991: 102. Postiglione, in nota alla sua edizione, riteneva "insostenibile" la lezione manoscritta, proponendo, poi, di "attenersi al testo vulgato" in quanto portatore di "un altro senso abbastanza soddisfacente"; Postiglione 1991: 132, n. 116.

22 Pohlenz 2001: 252.

${ }^{23}$ Bernardakis 1891: 275.

${ }^{24}$ Helmbold 1962: 320.

${ }^{25}$ Pohlenz 2001: 252.

${ }^{26}$ Amyot 1572: 89. Aulotte aveva notato come Amyot si fosse servito dell'aiuto occasionale delle traduzioni di Rousard e Xylander per rettificare errori ed inesattezze della tradizione manoscritta del De garrulitate plutarcheo; cf. Aulotte 1965: 202.

${ }^{27}$ Adriani 1827: 394. 
onorarla, e dirne bene”. Poi la traduzione latina fornita da Xylander ${ }^{28}$ è “... itaque fratrem honoret eique gratuletur", mentre la versio di Cruserius ${ }^{29}$ traduce “... $\&$ viri causa honores eam \& collaudes" e Rousard ${ }^{30}$ sceglie la traduzione “... uxorem vero fratris tanquam rem omnium sanctissimam mariti gratia suspicias, venereris \& collaudes".

Al passo in questione Schwartz e Pohlenz hanno ipotizzato la presenza di

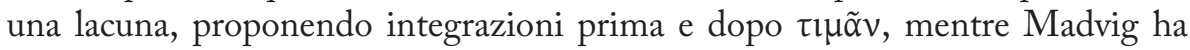

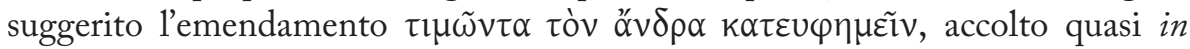
toto da Helmbold, ed a Postiglione è parsa in un primo luogo "insostenibile"

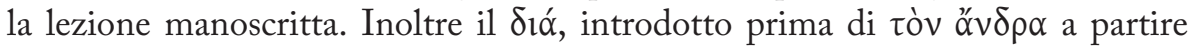
da Reiske, espunto da Dübner ${ }^{31}$, adottato da Bernardakis senza averlo visto nei propri codici, e che Postiglione non sapeva se ascrivere alla tradizione testuale o alla critica congetturale ${ }^{32}$, in realtà era una variante già presente e contrassegnata da un " $\Delta$ " ${ }^{33}$ a margine ${ }^{34}$ dell'Aldina $I, 23^{35}$, successivamente accolta nel testo dell'edizione francofortana del $1599^{36}$.

A questo punto sembra preferibile conservare la lezione dei codici, optando per una traduzione rispettosa del testo tràdito, come quella realizzata da Thomas Naogeorgus $^{37}$, che coglie nel segno anche sotto il profilo della comprensione effettiva del contenuto del passo plutarcheo. Traducendo "Mulierem autem fratris coniugem, ut omnium sacrorum sanctissimum, si aspicis aut colis, eius

${ }^{28}$ Xylander 1570: 439. Per quanto riguarda il De garrulitate plutarcheo, Aulotte sosteneva che Xylander avesse avuto sotto gli occhi il testo di Rousard, pur imitandolo in maniera libera e mai cieca; Aulotte 1965: 33.

${ }^{29}$ Cruserius 1573: 88.

${ }^{30}$ Rousard 1559: 33.

${ }^{31}$ Dübner 1856: 637.

${ }^{32}$ Postiglione 1991: 132-133, n. 116.

${ }^{33}$ Sul frontespizio dell'Aldina I,23 la nota autografa di Fulvio Orsini, tra l'altro, recita: " $\Delta$ littera Donati Poli codicem significat"; cf. Aldina 1509; Aulotte 1965: 180; Martinelli Tempesta 2006: 174. Questa segnalazione riconduce a Donato Polo (o Poli; morto verso il 1515 o 1516), professore di retorica e protetto di papa Leone X; cf. Negri 1722: 158; Aulotte 1965: 180. Per altre informazioni e indicazioni bibliografiche su D. Polo cf. Mercati 1908; Gaisser 1999: 317-318; Pontani 2000: 352, n. 63; Martinelli Tempesta 2006: 170 e n. 4. In mancanza del postillato autografo di Donato Polo, da considerarsi per ora perduto, si può ricostruire la consistenza originaria del gruppo di varianti di Polo (terminus ante quem per l'origine della raccolta è la data della morte di Polo stesso) soltanto fondandosi sul resoconto della raccolta di varianti di Leonico, Polo e Vettori realizzata da Donato Giannotti ed ampliata attingendo da materiale che circolava in ambienti dotti; cf. Martinelli Tempesta 2006: 170 e n. 4; 173-174; 180-181; 195-197.

${ }^{34}$ Cf. Aldina 1509: 497.

${ }^{35}$ Nota anche come Aldina "F. Orsini” e conservata presso la Biblioteca Apostolica Vaticana; cf. Aldina 1509; Martinelli Tempesta 2006: 172.

${ }^{36}$ Ed attribuita ad Amyot dall'apparato critico di Dumortier e dall'edizione di Postiglione; cf. Dumortier 1975: 171; Postiglione 1991: 102.

${ }^{37}$ Naogeorgus 1556: 132. 
virum honorare, deq; eo bene te loqui decet", si tratterebbe non di onorare e lodare la propria cognata solo a causa dell'affetto fraterno provato per il marito di lei, concetto che andrebbe a ricalcare il significato della proposizione precedente, ma di onorare e lodare il proprio fratello, marito di lei, quando ci si trova in presenza della cognata. Tale versio latina di Naogeorgus coglie in pieno il significato delle esortazioni di Plutarco, poiché, per difendere la concordia familiare, conviene sempre tessere le lodi del proprio fratello in presenza di sua moglie, al fine di evitare l'insorgere di discordie e di fomentare litigi. Infatti, nel momento in cui una critica fraterna dovesse trovare riscontro anche nelle perplessità della moglie di costui e, viceversa, qualora un biasimo della moglie dovesse trovare appoggio tra le riprensioni del cognato, la condivisione di un qualche punto di vista negativo tra cognati potrebbe forse generare forti dissidi tra moglie e marito.

Poi, quando Plutarco sostiene (479BC) che non bisogna muovere guerra a un proprio fratello, perché sarebbe un comportamento simile a quello dell'indovino di Arcadia che si fece fabbricare un piede di legno da sostituire al proprio, e conclude affermando che andare a cercare sostegno tra amici estranei alla famiglia sarebbe come:

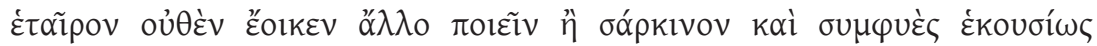

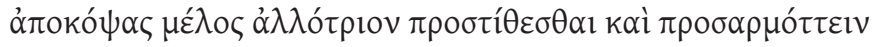

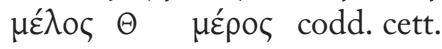

Naogeorgus traduce "nihil aliud facere videtur, quam carneam agnatamque sponte detruncare sibi partem, atque alienam apponere ac adaptare", mostrando di aver accolto la lectio $\mu \varepsilon ́ p o \zeta$, conservata dalla stragrande maggioranza dei testimoni del De fraterno amore, mentre Russardus opta per la resa "nihil aliud quam abscisso sponte membro carneo \& naturali alienum addere \& adaptare

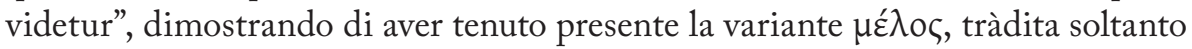
dalla famiglia di manoscritti denominata $\Theta^{38}$.

\section{Il DE FRATERNA beNEVOLENTIA Di LUdOVICUS RuSSARduS}

Nel cuore della Francia, nella regione storica del Berry, la città di Bourges (anticamente Biturgium) vide operare per alcuni lustri della seconda metà del 1500 Louis Rousard ${ }^{39}$ (nome latinizzato in Ludovicus o Lodoicus Russardus), giurista originario di Chartres divenuto Professeur presso l'ateneo cittadino

${ }^{38}$ I testimoni del De fraterno amore plutarcheo riconducibili alla famiglia $\Theta$ sono il cod. Marcianus Gr. 511 (saec. XIV), il cod. Ambrosianus Q 89 sup. (Gr. 689) (saec. XV), e il cod. Bruxellensis 18967 (ca. 1330); cf. Postiglione 1991: 37-38.

${ }^{39}$ Cf. anche Aulotte 1965: 32-33; 202-203; 205; 207-208; 332; 336. 
fondato dal re Louis XI nel 1464. La schola Biturgium era rinomata per la docenza accademica di François Douaren (o Duaren. Nome latinizzato in Franciscus Duarenus), giurista bretone di Moncontour allievo di Guillaume Budé a Parigi e del giureconsulto filologo milanese Andrea Alciato, esponente del movimento dottrinale della cosiddetta "Scuola culta", che si prefiggeva di rinnovare il diritto vigente all'epoca al fine di creare una "culta giurisprudenza" filologicamente coerente con le sue radici storiche giustinianee ${ }^{40}$.

Nel 1554 a Lione Louis Rousard aveva realizzato la traduzione latina del De garrulitate e del De esu carnium plutarchei per i tipi di Gryphius ${ }^{41}$, mentre nel 1559 pubblicò con privilegio reale a Parigi, presso l'editore Guillaume Morel, tipografo

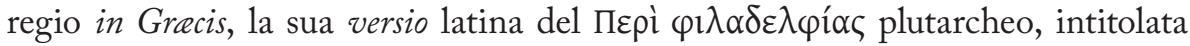
Plutarchi Charonei De amicitia fraterna ${ }^{42}$. Rousard compone la versio latina del De fraterno amore plutarcheo quale dono e segno di gratitudine e fedeltà ${ }^{43}$ alla famiglia degli Hurault, e si mostra lieto dell'approvazione ricevuta dal dedicatario, che qualifica la traduzione come opera "bene posita" e soddisfacente. Fin dall'epistola dedicatoria dell'opuscolo, datata alle idi di giugno del 1559 e indirizzata al "vir clarissimus" Robert Hurault ${ }^{44}$ (1483-1567), Ludovicus Russardus dichiara di aver tradotto in latino "ante quatuor annos" 45 il "Commentarius de Amicitia fraterna" plutarcheo proprio in quanto “... delectatus Plutarcho grauissimo \& doctissimo scriptore". L'autore afferma di aver comunicato la notizia della conclusione dell'opera versoria a tutti gli amici e, tra i primi, ad Adrien Tourneboeuf (Adrien Turnèbe o Adrianus Turnebus, alla latina), definito: “... non solum Galliae, sed etiam Europae totius ornamentum”. Se Turnebo fu avvisato solo ad opera terminata, come si deve ad un grande maestro verso cui si nutrono rispetto e timore reverenziale, Louis Rousard in una delle note conclusive ${ }^{46}$ della sua traduzione svela di aver usufruito di dotti consigli editoriali di due carissimi amici giuristi, consultati in merito ad una emendazione da apportare al testo

${ }^{40}$ E proprio nell'ottica di combattere la degradazione del corpus di istituti giustinianei, causata dall'infiltrazione sempre maggiore di glosse a detrimento della lettera del testo, va interpretata la collaborazione tra i giureconsulti filologi Louis Rousard e François Douaren per la pubblicazione a Lione, presso l'editore Rovillé, nel 1560, dello Jus civile; cf. Rousard 1560.

${ }^{41}$ Rousard 1554.

${ }^{42} \mathrm{Il}$ testo di riferimento è Rousard 1559.

${ }^{43}$ Rousard scriveva a Robert Hurault: “... ex hoc libello intelligere cupio me quicquid sum totum vestrae familiae perpetuo addictum fore, daturumque; operam, dum vita suppeditabit, ne meam in vos observantiam desideretis"; Rousard 1559: 4.

${ }^{44}$ Canonico, poi abate di Saint Martin d'Autun e titolare della carica di consigliere all'interno del Grand Conseil du Roi di Enrico II di Valois; successivamente, divenne cancelliere di Margherita di Navarra; cf. De Conihout 2007: 105-148.

${ }^{45}$ La stesura definitiva della traduzione latina di L. Rousard del De fraterno amore potrebbe dunque risalire al 1555.

${ }^{46}$ Cf. Rousard 1559: 36, n. 413. 
greco oggetto di traduzione. Quando, al capitolo XII (484C) dell'opuscolo, Russardus affronta il testo di una domanda rivolta da Pittaco al re dei Lidi, dopo aver apportato delle lievi modifiche alla punteggiatura dell'originale greco, annota: "Hic locus cum diu me torsisset, tandem ita emendandus esse mihi visus est. \& haec emendatio eo mihi magis probatur, quòd placet Eduardo Henrysio \& Adriano Puluæo, viris mihi amicissimis, iuris civilis, \& bonarum artium cognitione insignibus, quibuscum de ea communicaui”. Dunque i travagliati emendamenti di Rousard risultano rinsaldati dopo aver ricevuto l'approvazione ${ }^{47}$ di colleghi giuristi illustri conoscitori delle lettere antiche, quali il giudice scozzese Edward Henryson (latinizzato in Eduardus Henrysius, 15101590), docente di diritto romano a Bourges, collezionista e traduttore di testi classici che pubblicò nel 1555 a Lugdunum il Plutarchi Commentarius Stoicorum Contrariorum $^{48}$, e Adrien Poulvé (o Adrian Pulväus, latinizzato in Adrianus Pulucus), dottore in legge a Biturgium e autore di diversi trattati giuridici. Il De amicitia fraterna del docteur régent dell'Università di Bourges Ludovicus Russardus contiene poi una appendice finale "ad lectorem" che fornisce alcune precisazioni da cui si evincono interessanti dettagli inerenti al modus operandi del traduttore, e non solo. Quando afferma: "Non dubito quin ex collatione meae versionis cum exemplari Germanico, deprehendas me locis aliquot ab eo defecisse. Scias autem velim id consulto fecisse me, partim coniecturis gravibus ductum: quod sententiam aliter constare non posse iudicarem, partim fretum auctoritate codicis Francisci Duareni, Iuriconsultorum memoriae nostrae facilè principis, \& omni doctrinae genere exculti: quem plerunque secutus sum", l'autore lascia intendere di rivolgersi ad un pubblico piuttosto colto ed avvezzo alla filologia, che non solo avesse a disposizione un esemplare dell'edizione Basileense Frobeniana ${ }^{49}$, ma che

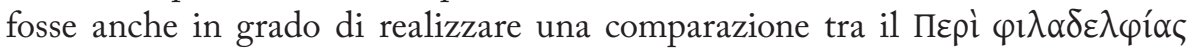
in essa stampato e la versio latina approntata da Rousard, rintracciando i loci ove il traduttore si fosse discostato dal testo greco. In secondo luogo Rousard, consapevole di essersi allontanato "in locis aliquot" dal testo della Frobeniana, qualifica le proprie scelte come deliberate e non casuali, rivendicando di aver agito seguendo un duplice tracciato: da un lato spinto da "graves coniecturae", giudicando che "... sententiam aliter constare non posse", e dall'altro lato sotto l'influenza della "auctoritas" di un imprecisato manoscritto, definito "codex

\footnotetext{
${ }^{47}$ Rousard afferma di aver portato a termine anche la traduzione del De garrulitate plutarcheo dopo aver ricevuto riscontro positivo da un dotto amico: “... adductus acri sinceroque iudicio carissimi viri Francisci Hospitalij Biturgium praefecti, qui dignum esse iudicat”; cf. Rousard 1554: 3 .

${ }^{48}$ Henryson 1555.

${ }^{49}$ L' "exemplar Germanicum" di cui parla Rousard è senza dubbio l'edizione Basileense dei Moralia plutarchei, in quanto le "emendationes \& variae lectiones" (e l'indicazione del preciso numero di pagina ove rinvenirle) citate successivamente dal traduttore lasciano identificare l'edizione di riferimento con la Frobeniana del 1542.
} 
Duarensis ${ }^{5_{0}}$ in quanto posseduto dal giureconsulto filologo François Douaren, il cui testo greco ammette di aver seguito nella maggior parte dei casi. Infine, ad uso del lettore ${ }^{51}$, Louis Rousard elenca una serie di quarantuno "emendationes \& variae lectiones", proponendo uno scrupoloso prospetto di singoli passi della editio Basileensis con la rispettiva lectio del "codex Duarensis" accolta in sede di traduzione o la eventuale modifica di punteggiatura proposta dal traduttore. In alcune delle note, poi, Russardus motiva anche le proprie scelte esprimendo dei giudizi di merito, a mo' di commento filologico ${ }^{52}$, stilistico e letterario, finalizzati di volta in volta a salvaguardare o restituire il "sensus" ${ }_{53}$, 1 '“elegantia" ${ }_{54}$ e 1'interpunctio" 55 del testo greco. Quindi si delinea il quadro di un giurista filologo, conscio delle capacità del proprio pubblico e convinto assertore delle proprie scelte, che per portare a termine la sua traduzione si avvale di un "codex" di riferimento, del proprio "iudicium" filologico e del supporto di fidati amici giureconsulti adusi a testi classici e manoscritti, premurandosi anche di fornire al lettore un breve ausilio esegetico atto a discernere e valutare in toto l'operato del traduttore.

La versio latina del De fraterno amore allestita da Louis Rousard è testimonianza di un intenso lavoro sul testo greco di Plutarco, che può risultare utile alla comprensione di passi lacunosi e molto discussi del trattato. In particolare, De frat. am. 485B8 esorta a valorizzare i fratelli, scegliendoli quali collaboratori o consiglieri per sottrarre campo all'invidia e alla discordia, presentan subito dopo una comparazione esplicativa del concetto sulla cui parte finale sono state formulate molte ipotesi. Nel dettaglio, dopo che il Cheronese ha suggerito di instaurare una positiva cooperazione fraterna

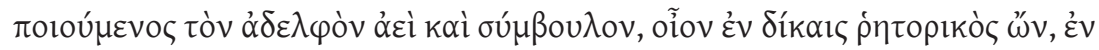

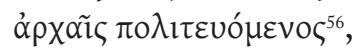

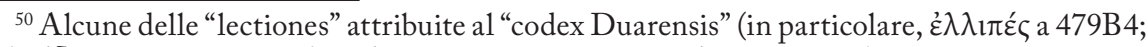

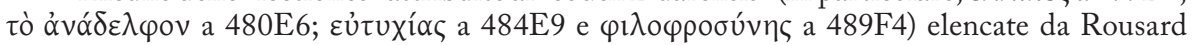
nella sezione "ad lectorem" potrebbero far identificare il manoscritto con il cod. Parisinus Gr. 1956 (med. s. XI); cf. Rousard 1559: 35-37.

51 “... ut de illis facilius statuere possis"; cf. Rousard 1559: 35.

${ }^{52}$ Cf Rousard 1559: 35, n. 411, dove il traduttore afferma: "Utraque lectio meo iudicio recipi posset”.

${ }_{53}$ Cf. Rousard 1559: 35, n. 411, dove Russardus annota: "Ex qua lectione sensus aptior elici mihi videtur".

${ }^{54}$ Cf. Rousard 1559: 36, n. 414, dove Rousard osserva: "Quam lectionem elegantiorem esse puto".

${ }_{55}$ Cf. Rousard 1559: 36, n. 412, dove il traduttore scrive: "Mendum hic obrepserat in interpunctione" e "Malim tollere interpunctionem".

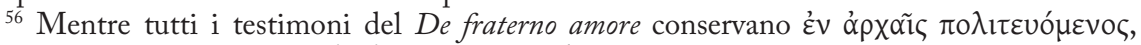

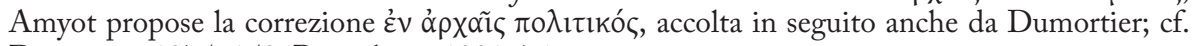
Dumortier 1975: 158; Postiglione 1991: 74. 
dove il palimps. Laurentianus 69,13 (saec. XIII) ed il cod. Parisinus Gr.

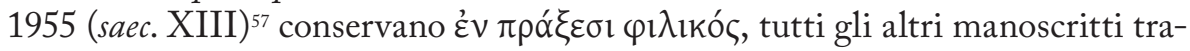

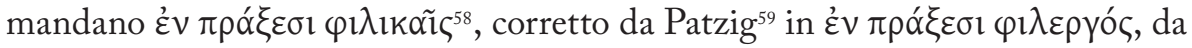

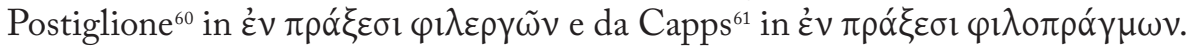
Pohlenz ${ }^{62}$, ritenendo il passo inemendabile, ha collocato una crux tra $\pi \rho \alpha ́ \xi \varepsilon \sigma l ~ e$ $\varphi \imath \lambda_{\imath \kappa \alpha i ̃}$ e, annotando in apparato critico "(sed $\varphi 1$ in $\alpha$ scr. rubr. in ras.)"63, ha

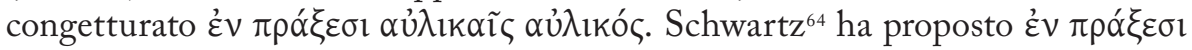

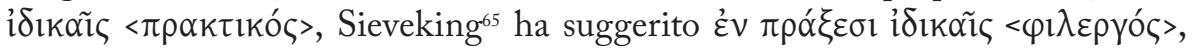

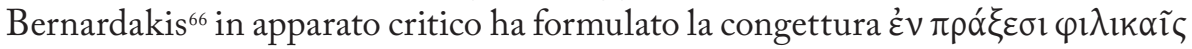
$\varphi \imath \lambda \imath \kappa o ́ s$, accolta anche da Dumortier ${ }^{67}$, ed Helmbold ${ }^{68}$, nell'apparato critico della

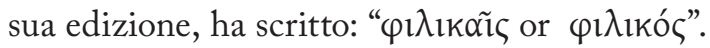

I traduttori hanno affrontato il passo in maniera differente, poiché Amyot ${ }^{69}$ ha tradotto "... s'il est homme qui ait beaucoup d'amis, en affaire”, Adriani ${ }^{70}$ “... se facendo profession d'amico, vuol seco trattare degli affari comuni" e Naogeorgus"1 "Qui verò fratrem in ijs, in quibus ipse praecellere videtur, adiutorem aut collegam assumit, aut consiliarium, veluti rhetoricus in causis,

${ }^{57}$ Aggiunto, però, da una seconda mano.

${ }^{58}$ Questa variante è accolta da Dübner (Dübner 1856: 588) e Bernardakis (Bernardakis 1891: 259). Anche Wyttenbach (Wyttenbach 1796: 975) optava per $\varphi \imath \lambda \iota k \alpha \tilde{\imath} \varsigma$, ma in apparato

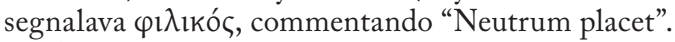

${ }^{59}$ Cf. Postiglione 1991: 74.

${ }^{60}$ Postiglione 1991: 74. In nota Postiglione ritiene che la struttura trimembre del periodo

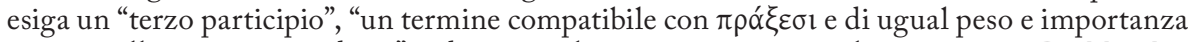
rispetto alle coppie precedenti" e di aver scelto un termine non solo presente in De lib. educ. 13A, ma che pare anche "soddisfacente da ogni punto di vista e non troppo lontano dalla tradizione manoscritta”; cf. Postiglione 1991: 125, n. 72. Tuttavia, la struttura non esige affatto un participio, mentre il termine prescelto da Postiglione non pare caratterizzato da tanto peso ed importanza, tantomeno se messo in parallelo con De lib. educ. 13A9.

${ }^{61}$ Congettura accolta nel testo critico di Helmbold; cf. Helmbold 1962: 286.

${ }^{62}$ Pohlenz 2001: 238.

${ }^{63}$ Stupisce il fatto che questo intervento sul codice $\alpha$ (si tratta del cod. Ambrosianus C 126 inf. (Gr. 859), risalente all' a. 1294), così importante per la tradizione di questo passo del De frat. am. e probabilmente alla base di molte delle emendazioni proposte dagli studiosi, non sia stato segnalato negli apparati critici precedenti all'edizione di Pohlenz.

${ }^{64}$ Cf. Pohlenz 2001: 238.

${ }^{65}$ Cf. Pohlenz 2001: 238.

${ }^{66}$ Bernardakis 1891: 259.

${ }^{67}$ Cf. Dumortier 1975: 158. In nota, poi, Dumortier precisava: "Nous croyons, pour notre part, qu'il y a eu haplologie et qu'il suffit pour trouver la bonne leçon de juxtaposer les deux

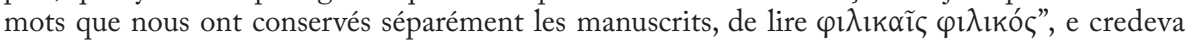
che Plutarco si riferisse forse "à un passage de la Cyropédie (VIII, 7, 15) où il est question de frères et d'amitié", in quanto "les amis sont précieux dans la vie politique. Ils confèrent du crédit et de la réputation”; cf. Dumortier 1975: 313, n. 1.

${ }^{68}$ Cf. Helmbold 1962: 286.

${ }^{69}$ Amyot 1572: 86.

${ }^{70}$ Adriani 1827: 376.

${ }^{71}$ Naogeorgus 1556: 116. 
rempublicam gerens in principatibus, amicisque; actionibus". La traduzione di Xylander ${ }^{72}$ è stata "... civilis rationis gnarus in rep. gerenda aut actionibus ad amiciciam pertinentibus", mentre quella di Cruserius ${ }^{73}$ "Velut orator in causis, qui remp. tractat, in magistratib. \& negociis amicorum, atque ut brevi dicam, nullo in negocio quod alicuius sit momenti”. Poi, più recentemente, Helmbold ${ }^{74}$ ha tradotto "... in practical affairs, himself being fond of such", Dumortier ${ }^{75}$ “... dans les affaires d'amitié s'il y est expert”, Aguilar ${ }^{76}$ “... en las empresas como una persona activa” e Postiglione ${ }^{77}$ “... negli affari, se è un uomo operoso”.

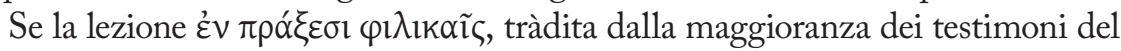
De fraterno amore ed accolta nelle traduzioni di Cruserius, Naogeorgus e Xylander, è stata quasi unanimemente riconosciuta dagli studiosi come una corruttela testuale, o per lo meno come un luogo problematico, la struttura tripartita del periodo ha di certo ispirato le congetture di Patzig, Capps e Postiglione e le traduzioni di Helmbold ed Aguilar, che risultano, però, in vario modo banali in quanto lessicalmente prigioniere della locuzione $\dot{\varepsilon} v \pi \rho \alpha ́ \xi \varepsilon \sigma l$ Troppo improntate alla giustapposizione

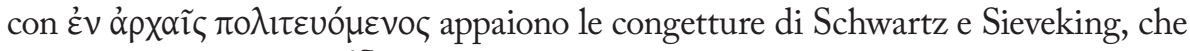

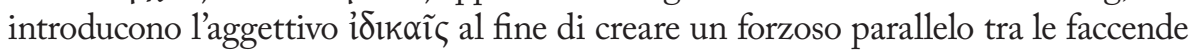
pubbliche e la sfera degli affari privati. A questo punto, se sembra ridondante la

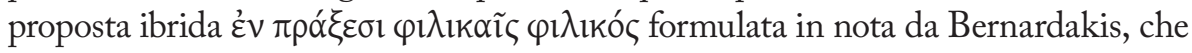
unisce per accumulazione le lectiones di tutti i manoscritti, pare preferibile la lezione

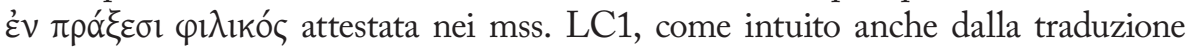
di Amyot, e soprattutto da quella di Louis Rousard ${ }^{78}$. Infatti, rendendo il discusso passo con la versio latina “... in actionibus, cum sit gratiosissimus", Russardus riesce a salvaguardare la struttura tripartita del periodo e propone di affiancarsi, negli affari così come nei processi e nelle cariche, un fratello di indole amichevole e ben incline alle relazioni interpersonali, qualità indispensabile per chi aspira a stringere relazioni proficue sotto il profilo degli scambi economici e commerciali.

Poi al dibattuto passo 484B10, dopo che Plutarco, posta la legislazione soloniana ad esempio di parità di trattamento ed avendo esortato a recepire le raccomandazioni di Platone in materia di uguaglianza all'interno della famiglia per mantenere la concordia tra fratelli, ha affermato:

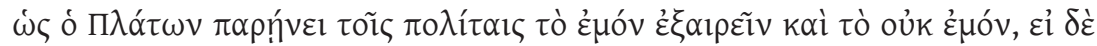

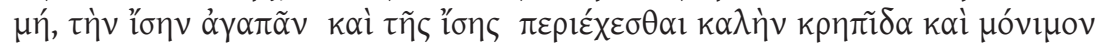
ónovoías

\footnotetext{
72 Xylander 1570: 434.

${ }^{73}$ Cruserius 1573: 84.

${ }^{74}$ Helmbold 1962: 287.

75 Dumortier 1975: 158.

${ }^{76}$ Aguilar 1995: 179.

77 Postiglione 1991: 75.

${ }^{78}$ Rousard 1559: 21.
} 


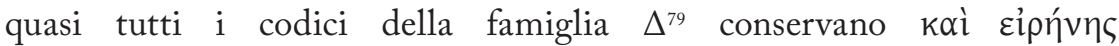

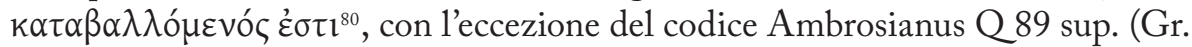

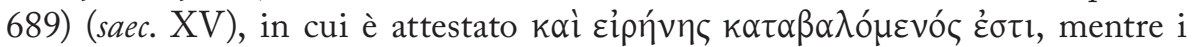

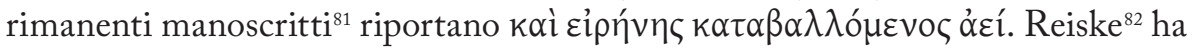

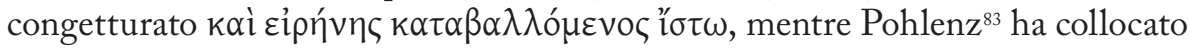

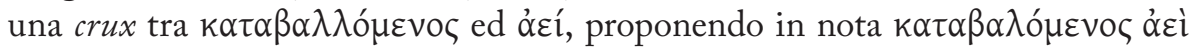

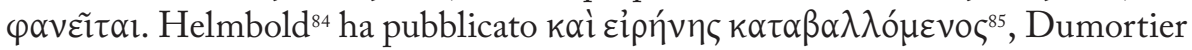
ha espunto $\alpha \varepsilon^{\prime}$ e ritenuto il passo lacunoso ${ }^{86}$ e Postiglione ha emendato il testo

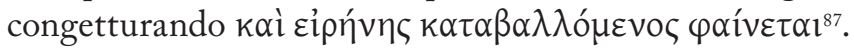

I traduttori hanno reso il testo in maniera molto varia, in quanto Amyot ${ }^{88}$ ha tradotto "certainement il asserroit un grand $\&$ beau fondement de paix, amitié \& concorde entre les freres" e Cruserius ${ }^{89}$ "bonum fundamentum pacis \& concordiae fecit". La traduzione di Adriani ${ }^{90}$ è stata “ ... gettò un bello e saldo fondamento d'unione e di pace" ${ }^{11}$ mentre quella di Xylander ${ }^{92}$ “... is egregium concordiae \& pacis fundamentum iaciens" ${ }^{93}$.

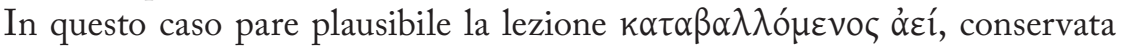
dalla stragrande maggioranza dei codici, dove il verbo $\kappa \alpha \tau \alpha \beta \alpha ́ \alpha \lambda \lambda \omega$ significa "stabilire, porre per fondamento", ricollegandosi alla terminologia legislativa

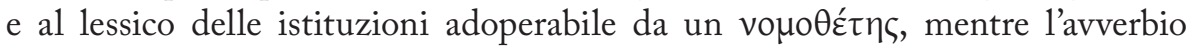
può tradurre sia nell'accezione di "costantemente" sia di "successivamente" un

${ }^{79}$ Che comprendono il cod. Parisinus Gr. 1956 (med. saec. XI) ed i codici della famiglia $\Theta$ (ovvero il cod. Marcianus Gr. 511 (saec. XIV), il cod. Ambrosianus Q 89 sup. (Gr. 689) (saec. XV), e il cod. Bruxellensis 18967 (ca. 1330); cf. Postiglione 1991: 37-38 e 70.

${ }^{80}$ Tale lezione è accolta da Wyttenbach, Dübner e Bernardakis; cf. Wyttenbach 1796: 971; Dübner 1856: 587; Bernardakis 1891: 257.

${ }^{81}$ Cf. Postiglione 1991: 37-38.

${ }^{82}$ Cf. Pohlenz 2001: 236.

${ }^{83}$ Pohlenz 2001: 236.

${ }^{84}$ Helmbold 1962: 280.

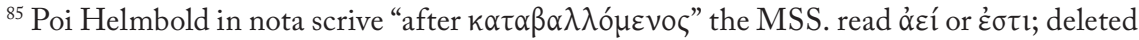
by W.C.H.”; cf. Helmbold 1962: 280.

${ }^{86}$ In particolare, in apparato critico ha scritto "lacunam suspicamur", ed in nota alla traduzione "... en jetant ainsi les beaux et durables fondements de la concorde et de la paix" ha ribadito il concetto, segnalando: "Le texte paraît ici lacunaire"; cf. Dumortier 1975: 155 e n. 3.

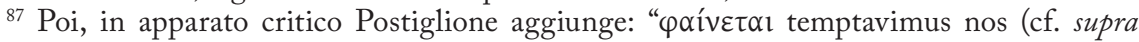

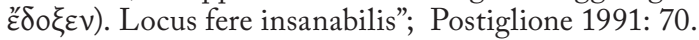

${ }^{88}$ Amyot 1572: 85.

${ }^{89}$ Cruserius 1573: 83.

${ }^{90}$ Adriani 1827: 374.

${ }^{91}$ Traduzione che ispira quella di Postiglione “... getta evidentemente un magnifico e saldo fondamento di concordia e di pace" (Postiglione 1991: 69; 71) e quella di Helmbold: “... and thus lays a fair and abiding foundation of concord and peace”, che in nota individua un probabile riferimento a Euripides, Phoenissae, 538 citate a 481A; cf. Helmbold 1962: 281.

92 Xylander 1570: 433.

93 Traduzione cui è improntata quella di Aguilar "colocando un hermoso y estable fundamento de concordia y paz"; Aguilar 1995: 177. 
significato coerente con il resto del periodo ed indirizzato alle prescrizioni politiche platoniche.

L'importanza attribuita ai codici della famiglia $\Delta$, che tramandano é $\sigma \tau l$, ha aperto la disputa che ha portato alle recenti congetture di Postiglione e Pohlenz, all'ipotesi di lacuna avanzata da Dumortier e all'espunzione operata da Helmbold. La probabile corruttela é $\sigma \tau \iota$, infatti, farebbe concludere il periodo in questione in una maniera tanto anomala da lasciar pensare ad una lacuna testuale, che diversi studiosi hanno provato in vario modo a colmare o spiegare.

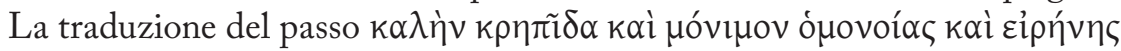

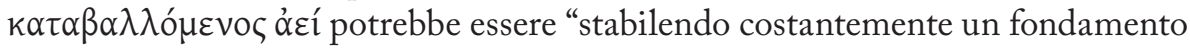
bello e saldo di concordia e pace", mentre le congetture ed integrazioni non sembrano imporsi per via della loro natura artificiosa e concettosa.

E proprio l'interpretazione di questo passo è affrontata con maggiore successo da Louis Rousard ${ }^{94}$, che ha tradotto “... iacto hoc egregio consensionis et concordiae fundamento", rendendo con l'ablativo assoluto latino un inciso sintattico che nel testo greco era formulato utilizzando il participio presente medio-passivo.

Naogeorgus ${ }^{95}$, invece, ha tradotto lo stesso passo “... is bonum concordiae fundamentum pacisque; iaciens, semper utatur exemplis illustribus", collegando $\kappa \alpha \tau \alpha \beta \alpha \lambda \lambda$ ó$_{\text {c }}$ vo al periodo successivo e ponendo prima di "iaciens" un punto e virgola quasi a mo' di cesura che, isolando la parola, ne rende piuttosto difficoltosa l'interpretazione nel contesto di riferimento.

\section{Il De fRAterna beneVolentia di Stephanus Niger}

Originario di Casalmaggiore, comune lombardo della provincia di Cremona, allievo di Demetrio Calcondila (1423-1511) e Ludovico Ricchieri $(1469-1525)^{96}$, il filologo Stefano Negri ${ }^{97}$ (nome latinizzato in Stephanus Niger) operò come professore di greco a Milano, dove si distinse per la pubblicazione della traduzione degli Heroica e delle Eikones di Filostrato di Lemno, del Sermo ad regem Syriae e del Quod principi aut regi philosophandum sit di Musonio di Tiro e della Consolatoria oratio ad Apollonium di Plutarco. Prima che la versio

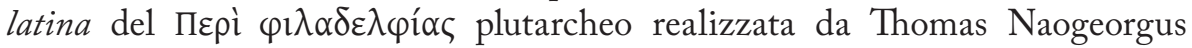
fosse definitivamente recepita ed accolta negli ultimi decenni del XVI secolo all'interno del II tomo della selezione di trattati dei Moralia tradotti in latino allestita nel 1572 da Stephanus ${ }^{98}$, e prima della pubblicazione del De fraterna

\footnotetext{
${ }^{94}$ Rousard 1559: 18.

${ }^{95}$ Naogeorgus 1556: 114.

${ }^{96}$ Umanista originario di Rovigo, noto anche con il nome umanistico di Celio Rodigino.

${ }^{97}$ Stefano Negri sarebbe vissuto tra il 1475 ed il 1540; cf. anche Aulotte 1965: 183.

${ }^{98}$ Cf. Stephanus 1572.
} 
benevolentia plutarcheo latinizzato da Ludovicus Russardus ${ }^{99}$, all'interno delle raccolte ${ }^{100}$ di traduzioni latine degli opuscoli plutarchei pubblicate a Basilea (nel 1518 presso uno stampatore ignoto ${ }^{101}$, nel 1530 presso l'officina di Cratander ${ }^{102} \mathrm{e}$ poi nel 1541 presso Isingrin ${ }^{103}$ ) e a Parigi (nel 1521 dai tipi di Badius Ascensius ${ }^{104}$, nel 1544 dalla stamperia di Vascosan e Roigny ${ }^{105}$, e nel 1566 presso gli stampatori Guillard e Belot ${ }^{106}$ e dalla stamperia di Macé ${ }^{107}$ ) comparve un opuscoletto latino di chiara ispirazione plutarchea intitolato De fraterna benevolentia ${ }^{108}$ ed attribuito a Stefano Negri. Spesso collocato, insieme al Commentarius in Plutarchi musicam di Carolus Valgulius, in coda ${ }^{109}$ ad ogni volume, soltanto nelle edizioni di Badius Ascensius, Cratander ed Isingrin il trattatello è preceduto da una breve epistola rivolta ai fratelli Antonin e Guillaume, figli di Antoine Duprat ${ }^{110}$, Gran Cancelliere del Regno di Francia per volere di Francesco I. Stefano Negri ricorda di aver promesso a suo tempo a Duprat padre di comporre un "opusculum de fraterna benevolentia" e di portarlo a termine al più presto "... ut amoris perpetuum esset monimentum" verso i due giovinetti che, seguendone i precetti elencati, avrebbero potuto conseguire una gloria imperitura presso i posteri. Conclusa l'epistola con la massima ovidiana che recita: fratrum quoque

${ }_{99}$ Pubblicazione che avverrà nel 1559 a Parigi, presso il tipografo regio A. Morel, con il titolo Plutarchi Charonei, De amicitia fraterna. Ludovico Russardo Iurisconsulto interprete; cf. Rousard 1559.

${ }^{100} \mathrm{Cf}$. anche Aulotte 1965: 326-337.

${ }^{101} \mathrm{Cf}$. Basilea 1518.

102 Cf. Cratander 1530.

${ }^{103}$ Cf. Isingrin 1541.

${ }^{104} \mathrm{Cf}$. Ascensius 1521.

${ }^{105}$ Cf. Vascosan 1544.

${ }^{106}$ Cf. Guillard 1566.

${ }^{107}$ Cf. Macaeus 1566.

${ }^{108}$ Più precisamente, il titolo dell'opera è Stephani Nigri De fraterna benevolentia libellum nella selezione di traduzioni latine di opuscoli plutarchei pubblicata nel 1518 a Basilea e Stephani Nigri De fraterna benevolentia opusculum nelle raccolte di opuscoli stampate da Badius Ascensius, Cratander, Vascosan e Roigny, Isingrin, Belot e Macé. Anche R. Aulotte indicava Stefano Negri tra i traduttori latini del De fraterno amore plutarcheo; Aulotte 1965: 332.

${ }^{109}$ In particolare, soltanto gli indici delle raccolte pubblicate da Badius Ascensius, Cratander e Isingrin fanno precedere i titoli dei due libelli di Negri e Valgulio dalla precisazione "Præterea, ne operum Plutarchi seriem turbaremus, sequentia in calcem reiecimus"; cf. Ascensius 1521; Cratander 1530; Isingrin 1541.

${ }^{110}$ Di famiglia nobiliare, divenuto vedovo si dedicò agli studi ecclesiastici divenendo prete, vescovo e cardinale. Presidente del Parlamento di Parigi (1508-1514), Cancelliere di Francia (1512-1513), Vescovo di Valence (1522), Arcivescovo Primate di Sens (1525-1535), Cardinale Presbitero di Sant'Anastasia per volere di Papa Clemente VII (1528-1535) e Legato Pontificio in Francia (1530), fu precettore del giovane duca Francesco d'Angoulême (futuro re Francesco I), redasse la raccolta di leggi civili chiamata Coutume d'Auvergne, negoziò il Concordato di Bologna (1516) con Papa Leone X ed ebbe un ruolo di primo piano durante la controversia tra il papato ed Enrico VIII d'Inghilterra sul divorzio. Sulla figura di Antoine Duprat cf. anche Albert-Buisson 1935. 
gratia rara est ${ }^{111}$, Stephanus Niger passa alla dedica ufficiale dell'opera agli stessi figli di Antoine Duprat, chiamato in causa quale "utriusque Galliae Magnus Cancellarius", appellativo che fornisce il terminus post quem per la redazione finale dell'opera ${ }^{112}$, a questo punto molto probabilmente terminata dopo che lo stesso Duprat, nell'anno 1519, aveva assunto anche il titolo di Cancelliere di Bretagna, cumulandolo con quello di Cancelliere del Regno di Francia, di cui era già stato insignito nel 1515. In un preambolo, Stephanus Niger cita l'aneddoto di Filostrato"13 , secondo cui la "benevolentia" tra Achille ed Elena, dopo la loro morte, spinse i "manes" dei due amanti ad incontrarsi su un'isola del Ponto e l'episodio, narrato da Carete di Mitilene e riferito da Ateneo ${ }^{114}$, secondo cui l'amore scoccato in una epifania notturna reciproca, portò Zariadre a sottrarre Odati al padre per sposarla. Tali esempi sono addotti per dimostrare come spesso anche solo l'ascolto della "virtus" e della "probitas" altrui, pur senza una visione diretta delle vicende riferite, possa ugualmente colpire e appassionare, creando una sorta di "consuetudo" tra l'ascoltatore e i fatti narrati. Il ragionamento si indirizza poi verso il filone encomiastico, in quanto l'autore sostiene di aver soltanto sentito parlare della "virtus" e "probitas" dei due figli di Antoine Duprat e, pur non avendoli mai visti di persona, confessa di essere divenuto talmente “... benevolentia prosequutus, quae me ad hoc de fraterna benevolentia opusculum ad Plutarchi aemulationem elaborandum adegerit", in modo da aiutare i fratelli Duprat ad accrescere l'affetto reciproco e a fornire loro un passatempo di lettura "haud molestum". Quindi Stefano Negri propone

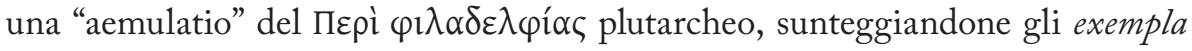
storici, mitologici e letterari e riproponendone i contenuti in forma abbreviata, costellata di proposizioni interrogative retoriche ${ }^{115}$ e connotata da un costante fervore omiliaco. In particolare, il De fraterna benevolentia di Negri riassume il testo plutarcheo rimescolandone i nuclei tematici e strutturandolo in modo da far prevalere il tono moralistico e le finalità pedagogiche a detrimento del tessuto argomentativo. Così i numerosi personaggi protagonisti degli aneddoti riferiti da Plutarco riappaiono in rapida successione, talora dentro eruditi quanto sbrigativi elenchi, mentre alcuni aneddoti plutarchei ricevono una attenzione maggiore

${ }^{111}$ Met. 1, 145.

$112 \mathrm{O}$, per lo meno, della redazione finale dell'epistola dedicatoria dell'opera. L' opuscoletto di Negri, infatti, fu pubblicato per la prima volta all'interno di una raccolta di trattati dei Moralia tradotti in latino nel 1518 a Basilea; cf. Basilea 1518.

${ }^{113}$ Cf. Philostr. Her. 54.

${ }^{114}$ Tale leggenda di origine scitico-persiana era molto popolare in Asia; cf. Athen. 13, $35=$ FGrHist 125 F 5.

${ }^{115}$ Particolarmente incalzanti le domande che fungono da reale incipit per l'opuscoletto: "Quid enim fraterna charitate praeclarius? Quid illustrius excogitari potest? Quid contra odio, simultate, seditioneque turpius, \& a quo magis ipsa abhorreat natura?”. La costante presenza di proposizioni interrogative a sostegno dell'argomentazione forse è mutuata dalla struttura degli opuscoli plutarchei dedicati alle Quaestiones. 
rispetto ad altri. Le citazioni letterarie di Sofocle, Euripide e Menandro inserite da Plutarco scompaiono; piuttosto Stephanus Niger preferisce ricordare la benevolenza fraterna di fama imperitura esemplificata dalla figura del cavaliere romano Proculo, citando i vv. 5-8 dell'Ode II di Orazio Flacco dedicata a Sallustio Crispo: Vivet extento Proculeius aevo / Notus in fratres animi paterni: / Ilium aget penna metuente solvi / Fama superstes?

La consuetudine con la lingua ellenica di Stefano Negri docente di greco emerge quando, discutendo a proposito del custodire buoni rapporti con i fratelli nel contesto della innata propensione umana alle relazioni sociali, scrive "Ipsa nanque amicitiarum ac consuetudinum innata nobis cupiditas maximo esse documento debet, ut fratres observemus ac colamus, eorumque amicitiam in

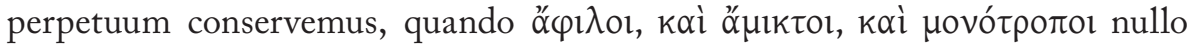
pacto vivere possemus" e quando, elogiando le prerogative della famiglia Duprat, cui appartenevano i dedicatari dell'opuscoletto, afferma: "Quid de parentibus loquar, quorum alterius quidem tam variae ac multiplices sunt virtutes, ut quum

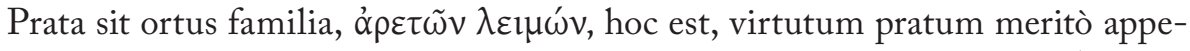
llari possit". Poi l'opera di Stefano Negri, dopo uno sperticato elogio di Antoine Duprat, definito "utriusque Galliae oraculum", pari ad Aristide e Radamanto in "prudentia" ed "aequitas", e a Gorgia per "facundia", si conclude invitando i fratelli Antonino e Guglielmo a rispettare i precetti esposti nel trattatello per preservare la "charitas fraterna" da futili amicizie esterne e degenerazioni, e mantenere saldo il "summum decus" familiare nel tempo presso conoscenti e sconosciuti.

Dunque negli anni delle prime raccolte di opuscoli dei Moralia plutarchei tradotti in latino da intellettuali di tutta Europa, in assenza di una completa ed

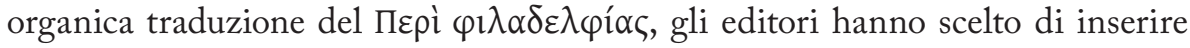
nella lista delle versiones latinae anche questo opuscoletto di Stefano Negri, compendio senza troppe pretese del De fraterno amore contenente diverse tracce del pensiero plutarcheo ridotte ad esile ossatura erudita di un'opera completamente differente. Tale trattatello, comparso per la prima volta nel 1518 a Basilea, non è che la prima testimonianza in ordine cronologico del successo riscosso nell'Europa del XVI secolo dal De fraterno amore plutarcheo, opuscolo successivamente latinizzato da Thomas Naogeorgus (1556) e Ludovicus Russardus (1559), a riprova di un interesse sempre vivo per la tematica trattata, le vicende narrate e la sensibilità letteraria di un autore greco la cui opera è stata ritenuta meritoria della più ampia attenzione e diffusione per via di una portata culturale di estrema attualità e quale conseguenza di un contenuto fruibile da lettori intellettuali e non. 


\section{Bibliografia}

\section{Edizioni}

Adriani 1827 = Opuscoli di Plutarco volgarizzati da Marcello Adriani, nuovamente confrontati col testo e illustrati con note da Francesco Ambrosoli. Tomo Terzo. Milano.

Aguilar 1995 = Plutarco. Obras Morales y de Costumbres (Moralia) VII. Introducciones, Traducciones y Notas por Rosa María Aguilar. Editorial Gredos. Madrid.

Aldina 1509 = Plutarchi Opuscula LXXXXII. Index Moralium omnium, et eorum quae in ipsis tractantur, habetur hoc quaternione. Venetiis.

Amyot $1572=$ Les Oeuvres Morales et meslées de Plutarque, Translatées de Grec en François par Messire Iacques Amyot, à present Euesque d'Auxerre, Conseiller du Roy en son privé Conseil, E̋ grand Aumosnier de France. Tome Premier. A Paris, De l'Imprimerie de Michel de Vascosan, avec Privilege du Roy.

Ascensius 1521 = Opuscula Plutarchi Charonei sedulo undequaque collecta, E。 diligenter recognita, ac in unam faciem bellatule coimpressa: quorum ante prefationem patebit E numerus E' series, praemisso q. amplissimo E rerum E verborum indice. Vaenundantur in Officina Ascensiana.

Basilea 1518 = Plutarchi Charonensis opuscula quadam. Des. Erasmo Roterodamo, Stephano Nigro, Angelo Barbato, Bilibaldo Pirckheymero, E Philippo Melanchthone Brettano, interpretibus. Quorum catalogum sequentis pagelle elenchus indicabit. Apud inclytam Basileam.

Basilea 1542 = Plutarchi Moralia opuscula, multis mendarum milibus expurgata, Basileae, per Hier. Frobenium et Nic. Episcopium.

Bernardakis 1891 = Plutarchi Chaeronensis Moralia, recognovit G.N. Bernardakis, vol. III, Lipsiae.

Cratander 1530 = Plutarchi Charonei, philosophi historicique clarissimi, Opuscula (quae quidem extant) omnia, undequaque collecta, Eo diligentissime iampridem recognita. Quorum catalogum mox versa pagina indicabit. Cum amplissimo E rerum E verborum indice. Basileae, in officina And. Cratandri.

Cruserius 1573 = Plutarchi Chaeronei, Ethica, sive Moralia, Opera quae extant, omnia: Interprete Hermanno Cruserio I.C. atque illustrissimi Ducis Cliuensis E' Iuliacensis Consiliario. Accesserunt rerum E verborum fidelissimi indices. Cum gratia et priuilegio Regio, Basileae, apud Thomam Guarinum.

Dübner 1856 = Plutarchi Scripta Moralia, ex codicibus quospossidet Regia Bibliotheca omnibus ab Kóv $\omega$ cum Reiskiana editione collatis emendavit F. Dübner. Graece et Latine. Volumen Primum, Parisiis.

Dumortier 1975 = Plutarque, Euvres morales, tome VII, première partie (traités 27-36). Texte établi et traduit par Jean Dumortier avec la collaboration de 
Jean Defradas. Collection des Universités de France, Paris, Belles Lettres. Guillard 1566 = Plutarchi Charonei Philosophi atque Historici clarissimi Moralia Opuscula, quotquot reperire licuit Latio donata: Non a peregrine modo, sed a nostra quoque, hoc est Christiane philosophice candidatis omnibus serie ac sedule legi perdiscique dignissima. Parisiis. Apud Gulielmum Guillard, \& Thomam Belot in via Iacobæa, sub D. Barbarae signo.

Helmbold 1962 = Plutarch's Moralia in Fifteen Volumes. Volume VI (439A-523B) with an English Translation by W.C. Helmbold. Cambridge, Massachusetts/ London.

Henryson 1555 = Plutarchi Commentarius Stoicorum Contrariorum, Ed. Henrysone Iureconsulto interprete, ad Franciscum Duarenum Iureconsultorum aetate sua principem. Lugduni, apud Gulielmum Rovillium, sub scuto Veneto.

Isingrin $1541=$ Plutarchi Charonei philosophi et historici clariss. Opera Moralia, quae in hunc usque diem latine extant, uniuersa. Huius viri singularem disciplinarum omnium scientiam, E exquisitam rerum varietatem, solide eruditionis studioso notiorem esse decet, quam ut encomium eius aliquod hic expectet. Bibliothecam enim totam ecquis laudarit satis. Librorum catalogum sequens pagina monstrabit. Cum amplissimo rerum Ẽ verborum Indice. Sub Augustiss. Imperatoriæ Maiest. Edicto \& privilegio. Basileae, apud Mich. Isingrinium.

Macaeus 1566 = Plutarchi Charonei Philosophi atque Historici clarissimi Moralia Opuscula, quotquot reperire licuit Latio donata: Non a peregrine modo, sed a nostra quoque, hoc est Christiance philosophice candidatis omnibus serie ac sedule legi perdiscique dignissima. Parisiis. Apud Ioannem Macæum, sub scuto Britaniæ in monte D. Hilarij.

Naogeorgus 1556 = Plutarchi Chaeronensis, Summi Philosophi, Libelli septem, in Latinum conversi, cum antea versi non essent: Quorum catalogum versa pagina invenies. Thoma Naogeorgo Straubigensi interprete. Basileae, per Ioannem Oporinum.

Pohlenz 2001 = Plutarchus Moralia vol. III, recensuerunt et emendaverunt W.R. Paton-M. Poblenz-W. Sieveking, editio stereotypa editionis primae (MCMXXIX). Monachii et Lipsiae.

Postiglione 1991 = Plutarco. L' amore fraterno/L' amore per i figli. Introduzione, testo critico, traduzione e commento a cura di A. Postiglione. Napoli, M. D'Auria.

Rousard 1554 = Plutarchi Chaeronei Philosophi Clarissimi Liber de garrulitate. Eiusdem De esu carnium orationes duae, Lodoico Russardo interprete. Apud Seb. Gryphium. Lugduni.

Rousard 1559 = Plutarchi Charonei, De amicitia fraterna. Ludovico Russardo Iurisconsulto interprete. Parisiis. Apud Guil. Morelium, in Græcis typographum Regium. Ex privilegio Regis. 
Rousard 1560 = Jus civile, manuscriptorum librorum ope, summa diligentia $\mathcal{E}^{\circ}$ integerrima fide infinitis locis emendatum, Eं perpetuis notis illustratum, $L$. Russardo J.C. antecessore in celeberrima Biturgium schola auctore: Consilio tamen et auctoritate Fran. Duareni J.C. qui summaria in Pandectas praescripsit, novellasque constitutiones ad optimum Graecum exemplar collatas, antiquae integritati restituit. Caetera utraque praefatio te docebit. Cum privilegio. Lugduni, apud G. Rouillium.

Stephanus 1572 = Plutarchi Cheronensis opuscula varia: qua magna ex parte sunt philosophica: Vulgo autem Moralia opuscula nimis angusta appellatione vocantur. Ex diversorum interpretationibus quarum quadam sunt Henrici Stephani, non antea edita. Indici superioribus multo locupletiori adiuncta sunt Annotationes eiusdem Henr. Stephani. Excudebat Henr. Stephanus. Cum privilegio Caes. Maiestatis, et Christianiss. Galliarum Regis.

Vascosan 1544 = Plutarchi Charonei, Philosophi \& Historici gravissimi, Ethica, seu Moralia opuscula, quae quidem in hunc usque diem è greco in latinum conuersa extant, uniuersa: Quorum catalogum proxima post indicem pagina monstrabit. Parisiis. Imprimebat Michaël Vascosanus sibi, \& Iohanni Roigny. Cum privilegio.

Xylander 1570 = Plutarchi Chaeronensis Moralia, quae usurpantur. Sunt autem omnis Elegantis doctrinae Penus: Id est, varij libri: morales, historici, physici, mathematici, denique; ad politiorem litteraturam pertinentes et humanitatem: omnes de Graeca in Latinam linguam transcripti summo labore, cura, ac fide: Guilielmo Xylandro Augustano interprete. Accesserunt his indices locupletissimi. Basileae, per Thomam Guarinum.

Wechel 1599 = Plutarchi Chaeronensis quae exstant omnia, cum Latina interpretatione Hermanni Cruserij: Gulielmi Xylandri, et doctorum virorum notis, et libellis variantium lectionum ex Mss. Codd. diligenter collectarum, et indicibus accuratis, t. II, Francofurti, apud Andreae Wecheli heredes, Claudium Marnium, \& Ioannem Aubrium.

Wyttenbach 1796 = Plutarchi Chaeronensis Moralia, id est opera, exceptis Vitis, reliqua. Graeca emendavit, notationem emendationum, et Latinam Xylandri interpretationem castigatam, subjunxit, animadversiones explicandis rebus ac verbis, item indices copiosos, adjecit D.A. Wyttenbach, tom. II, pars II. Oxonii, e typographeo Clarendoniano.

\section{Altri studi}

Albert-Buisson, F. (1935), Le Chancelier Antoine Duprat, Paris.

Aulotte, R. (1965), Amyot et Plutarque, La Tradition des Moralia au XVIe Siècle, Genève.

Brokate, K. (1913), De aliquot Plutarchi libellis, diss. inaug., Gottingae. 
De Conihout, I. (2007), "Jean et André Hurault: deux frères ambassadeurs à Venise et acquéreurs de livres du cardinal Grimani”, Italique 10: 105-148.

De Romilly, J. (1979), La douceur dans la pensée grecque, Paris.

Gaisser, J. H. (1999), Pierio Valeriano on the ill fortune oflearned men. A Renaissance bumanist and his world, Ann Arbor, The University of Michigan Press.

Häberlein, M. (2012), The Fuggers of Augsburg: Pursuing Wealth and Honor in Renaissance Germany, University of Virginia Press.

Hübner, A. (1913), "Studien zu Naogeorgus”, Zeitschrift für deutsches Altertum 54: 297-338.

Hübner, A. (1920), "Studien zu Naogeorgus”, Zeitschrift für deutsches Altertum 57: 193-222.

Irigoin, J. (1987), Histoire du texte des Euvres morales de Plutarque, in R. Flacelière-J. Irigoin-J. Sirinelli-A. Philippon (eds.), Plutarque, Euvres morales, t. I.1. Paris, 226-324.

Jones, C.P. (1966), “Towards a chronology of Plutarch's works”, JRS 56: 61-74.

Kluger, M. (2009), Die Fugger. Die deutschen Medici in und um Augsburg, Augsburg.

Krojer, F. (2006), Aufschluss des Gäubodens, München.

Levinger, H. (1935), "Die Bühne des Thomas Naogeorgus", Archiv für Reformationsgeschichte 32: 145-166.

Martinelli Tempesta, S. (2006), Studi sulla tradizione testuale del De tranquillitate animi di Plutarco, Firenze.

Mercati, G. (1908), “A proposito di un'oscura sottoscrizione”, RhM 64: 322-325.

Negri, G. (1722), Scrittori fiorentini, Ferrara.

Pontani, A. (2000), "Postille a Niccolò Tomeo e Giovanni Ettore Maria Lascaris", Bollettino della Badia greca di Grottaferrata 54: 337-368.

Roloff, H.G. (1979), "Problem von Humanismus und Reformation", in A.A.V.V. (eds.), L'Humanisme Allemand 1480-1540. XVIIe Colloque International de Tours. München/Paris, 455-475.

Shade, O. (1863), Satiren und Pasquille aus der Reformationszeit, Hannover.

Strieder, J. (1931), Jacob Fugger the Rich: Merchant and Banker of Augsburg, 14591525, New York.

Theobald, L. (1931), "Zur Lebensgeschichte des Thomas Naogeorgus", Zeitschrift für Bayrische Kirchengeschichte 6: 143-165.

Treu, M. (1873), Der sogenannte Lampriascatalog der Plutarchschriften, Progr. Waldenburg in Schlesien.

Volpe Cacciatore, P. (2010), "La traduzione latina del Naogeorgus del De genio Socratis", in L. Van Der Stockt-F. Titchener-H.G. Ingenkamp-A. Pérez 
Il De fraterno amore di Plutarco tra Thomas Naogeorgus, Ludovicus Russardus e Stephanus Niger

Jiménez (eds.), Gods, Daimones, Rituals, Myths and History of Religions in Plutarch's Works. Studies devoted to Professor Frederick E. Brenk by the International Plutarch Society. Málaga/Logan, 459-466.

Ziegler, K. (1965), Plutarco, ediz. it. a cura di B. Zucchelli, trad. it. di M. R. Zancan Rinaldini, Brescia. 